\title{
Wavelength spacing tenable capability of optical comb filter using Polarization Maintaining Fiber
}

\author{
J. N. Sikta ${ }^{1}$, M. S. Islam ${ }^{1}$, O. F. Rasel ${ }^{2}$, and N. N. Ripa ${ }^{1}$ \\ ${ }^{l}$ Department of physics, Jahangirnagar University, Savar, Dhaka-1342, Bangladesh \\ ${ }^{2}$ Physics Discipline, Khulna University, Khulna
}

\begin{abstract}
In this paper, tuneable comb filter using single-mode fiber (SMF)has been proposed and theoretically studied its capability of tuning the output spectral spacing continuously by using the birefringence property of polarization maintaining fiber (PMF). The proposed filter consists of one $Q W P$, one HWP, one SMF and one PMF segment. Depending on the dynamic settings of wavelength of the input signal, refractive index, and length of the fiber material the comb filter offers the continuous channel spacing tunability. Obtained output spectra are quite similar as calculated from equation. Channel spacing can be tuned at any desired value with the help of this comb filter. PMF based filter is very useful for long distance transmission.
\end{abstract}

Keywords: Comb filter, Spectral spacing, SMF, PMF, tunability.

\section{Introduction}

Fiber optic communication systems have become the effective technology for high speed information transmission medium in both the long haul systems and the local and metropolitan networks. Within the last twenty years, fiber based devices have been developed extensively in the use as filters, sensors, amplifiers and lasers.The filter is an important component in the fields of fiber optic communication and sensing applications. The birefringent comb filter plays a significant role in the processing of microwave and optical signal. Because of the increasing application in multi wavelength fiber lasers[1], it has attracted much attention in recent years [2]. It is desirable for a comb filter to be tunable in spectral spacing.

Various types of filters such as Fabry-Perot filter [3], Lyot birefringent filter [4], fiber grating filter [5], birefringence fiber filter [6], Sagnac loop interferometer [7], Mach-Zehnder filter [8], and tunable optical comb filters [9] have been proposed. All the fiber filters have the advantages of low insertion loss, low cost, and good compatibility in the fiber communication system. Waveguide-based MZI as a comb-like filter is a better choice [10] because of its inherent advantages of insensitivity to environmental change and high reliability. Recently, polarization independent tunable all-fiber comb filter based on a modified dual-pass MZI has been reported [11]. But this comb filter can be tuned for two fixed spectral spacing. Still we are far behind from the continuous tunability of the spectral spacing and the simplicity of maintaining polarization.

Recently, polarization maintaining fiber has drawn much attention in the field of fiber optic communication for its higher birefringence property. Research efforts in optical device technology have led to the emergence of polarization maintaining fiber devices that promise to reduce the maintenance cost and flexibility.We have introduced a new architecture of optical comb filter based on Mach-Zehnder interferometer which provides the highest tunability of comb spacing. In this paper, the design for a tunable comb filter based on MZI has been proposed and investigated theoretically. In a comparison, the insertion loss in optical fiber MZI tunable comb filter is very low and the extinction ratio is high as compared to the ordinary MZI filter. These types of filter are very simple in design and expected to have more efficient and flexible in functionality.

\section{Methodology}

The theoretical model of the proposed tunable optical comb filter is developed based on the schematic diagram as shown in Fig 1 .

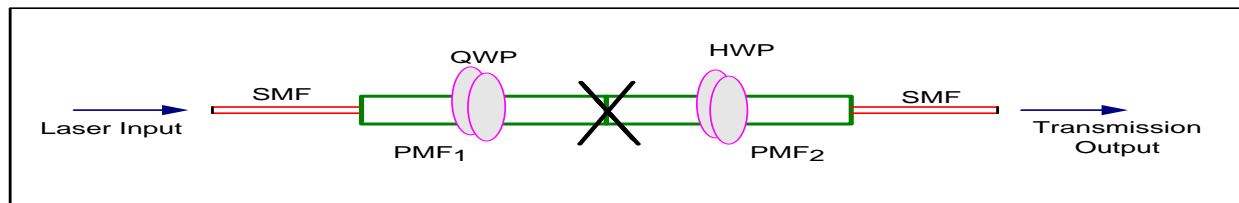

Fig 1: Schematic of proposed tunable optical comb filter.

The structure consists of one QWP, one HWP, one SMF, and one PMF segment. Two PMF lengths are considered to splice together in between two SMF segments. The splicing points are shown by a cross symbol in the figure. When light enters through the SMF it splits into two parts inside the PMF. One is along slow axis and 
another is along fast axis. Due to the path difference between slow and fast axis, there is a discrete path difference between two parts of the signal. This path difference can be controlled with the rotation of QWP. HWP is usually used to change the effective length of the PMF segment from maximum to minimum. But for the simplicity of the calculation HWP is considered fixed at $90^{\circ}$ and here QWP is kept fixed at $45^{\circ}$. When these two parts of signal enter into the SMF again interference occurs. As a result of interference, sinusoidal transmission output is supposed to be expected. The spectral spacing of the output depends on length, refractive index of the fiber material and the wavelength of the input signal. Peak wavelength position of the output transmission also depends on these factors.

For the proposed comb filter, we can write the expression,

$$
\left|\frac{\text { Eout }}{E_{\text {in }}}\right|=[F 1][P][Q W P][H W P][F 2]
$$

The matrices of the two interferometer arms, $\left[F_{n}\right](\mathrm{n}=1,2)$ are

$$
\begin{aligned}
& {\left[F_{1}\right]=\left[\begin{array}{cc}
e^{j k n_{x} \mathrm{~L}} & 0 \\
0 & e^{j k n_{y} \mathrm{~L}}
\end{array}\right]} \\
& {\left[F_{2}\right]=\left[\begin{array}{cc}
e^{j\left(k n_{x} \mathrm{~L}+\varphi\right)} & 0 \\
0 & e^{j\left(k n_{y} \mathrm{~L}+\varphi\right)}
\end{array}\right]}
\end{aligned}
$$

where $\varphi$ is the phase difference due to path difference between two interferometer arms.

$\mathrm{P}$ is the polarization element of the fiber and is expressed as,

$$
[P]=\left[\begin{array}{cc}
\cos \theta & -\sin \theta \\
\sin \theta & \cos \theta
\end{array}\right]
$$

QWP with fast axis vertical is given by;

$$
\mathrm{QWP}=\left[\begin{array}{cc}
\left(\cos \frac{2 \pi}{4}+i \sin \frac{2 \pi}{4}\right) & 0 \\
0 & -i\left(\cos \frac{2 \pi}{4}-i \sin \frac{2 \pi}{4}\right)
\end{array}\right]
$$

HWP with fast axis vertical is given by;

$$
\mathrm{HWP}=\left[\begin{array}{cc}
\left(\cos \frac{2 \pi}{2}+i \sin \frac{2 \pi}{2}\right) & 0 \\
0 & -i\left(\cos \frac{2 \pi}{2}-i \sin \frac{2 \pi}{2}\right)
\end{array}\right]
$$

Using equations (2-6) in equation (1) we have,

$$
\left|\frac{\text { Eout }}{E_{\text {in }}}\right|=-\frac{1}{\sqrt{2}} \cos k n_{x} L \cos k n_{y} L \cos \left(k n_{x} L+\emptyset\right) \cos \left(k n_{y} L+\emptyset\right)
$$

Now, transmission, $\mathrm{T}=\left|\frac{\text { Eout }}{E_{\text {in }}}\right|^{2}$

$$
=\frac{1}{2}\left[\cos k n_{x} L \cos k n_{y} L \cos \left(k n_{x} L+\emptyset\right) \cos \left(k n_{y} L+\emptyset\right)\right]^{2}
$$

When, $\varnothing=90^{\circ} ; \quad$ Equation (7) gives,

$$
\mathrm{T}=\frac{1}{32}\left[\sin ^{2} 2 A\right] ; \text { when } n_{x}=n_{y}=n \text {, we have, } A=B=k n L
$$

When, $\varnothing=45^{\circ}$ or $0^{\circ}$; Equation (7) gives,

$$
\mathrm{T}=\frac{1}{2} \cos ^{8}(k n L) ; \text { when } n_{x}=n_{y}=n
$$

Equations (8) and (9) give output transmission spectra.

Proposed filter has the comb spacing $(\Delta \lambda)$ given by the equation,

$$
\Delta \lambda=\frac{\lambda^{2}}{\Delta n L}
$$

Equation (10) indicates that spectral spacing depends on the refractive index (n), and length (L) of the fiber material. It also depends on the wave vector $(k)$ i.e. wavelength $(\lambda)$ of the input signal. 


\subsection{Transmission}

\section{Results And Discussions}

Equations (8) and (9) are obtained as transmission equations of the proposed tunable optical comb filter. The specific values of different parameters have been taken into account to calculate the output transmission and obtained transmission spectra are shown in the figures 2 and 3.

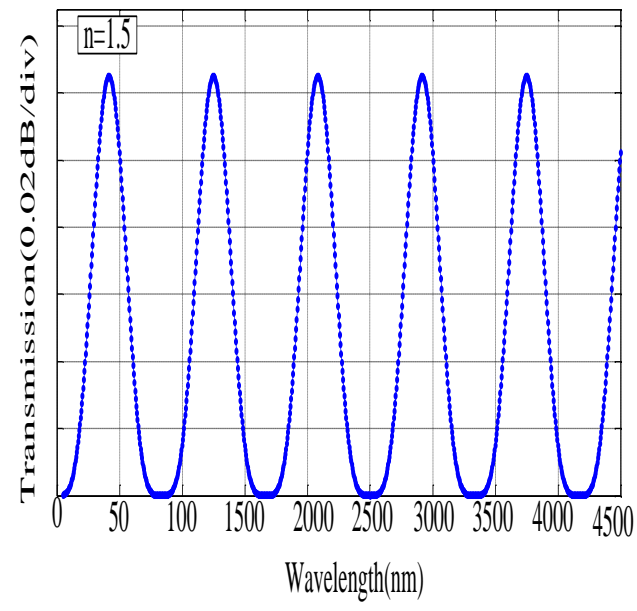

(a)

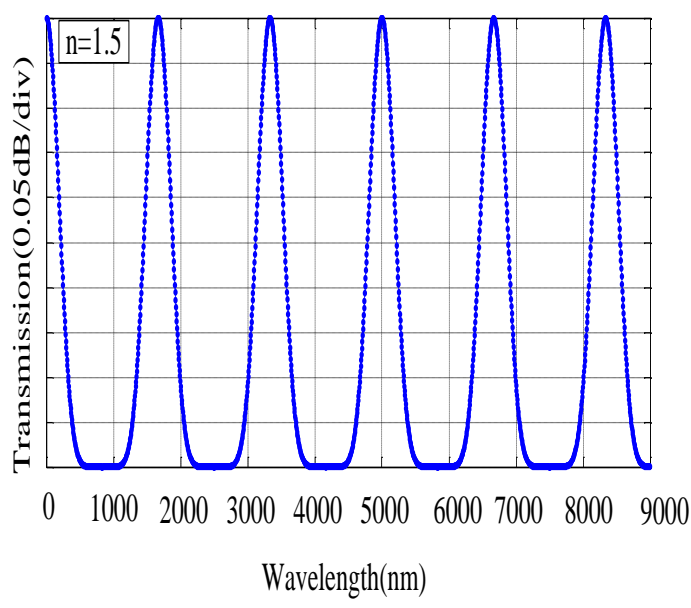

(b)

Fig 2: Output transmission spectra with the variation of wavelength (a) $\emptyset=90^{\circ}$;(b) $\emptyset=45^{\circ}$ or $0^{\circ}$

Fig. 2(a) represents the perfect sinusoidal curve. This transmission curve is drawn based on the equation (8). Fig. 2(b) represents the output transmission spectra according to theequation (9). In both cases,expected output transmissions have been obtained.

\subsection{Spectral Spacing}

One of the main objectives to design the comb filter is to change the spectral spacing. Spectral spacing can be changed with the variation of different parameters. Here, wavelength of the input laser signal, refractive index and length of the fiber material have been varied to change the spectral spacing in the output transmission spectra of equations (8) and (9).

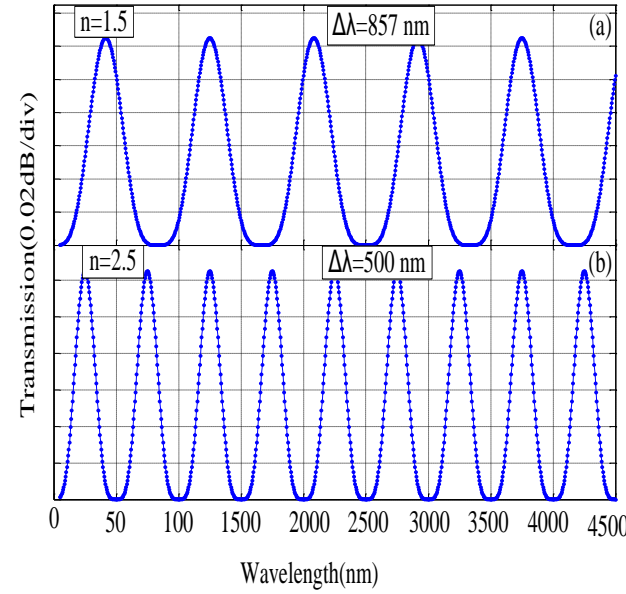

(a)

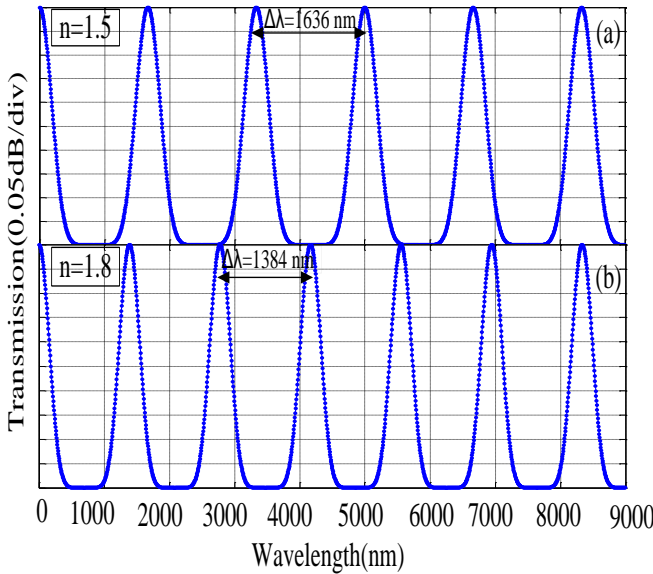

(b)

Fig 3: Transmission spectra for the variation of wavelength. (a) $\varnothing=90^{\circ}$; (b) $\varnothing=45^{\circ}$ or $0^{\circ}$

Figures 3(a) and 3(b) represent the transmission spectrum with the variation of wavelength based on equation (8) and (9). In fig. 3(a), the calculated spectral spacings are found to be $857 \mathrm{~nm}$ and $500 \mathrm{~nm}$ when the refractive indices are 1.5 and 2.5 respectively. In fig. 3(b), spectral spacing changes from $1636 \mathrm{~nm}$ to $1384 \mathrm{~nm}$ for the change of the refractive index from 1.5 to 1.8 . So it is observed that in both cases, the spectral spacing decreases with the increase of refractive index of the fiber material. Thus we can achieve required number of channels by increasing or decreasing the refractive index of fiber material i.e. using graded-index fiber materials. 


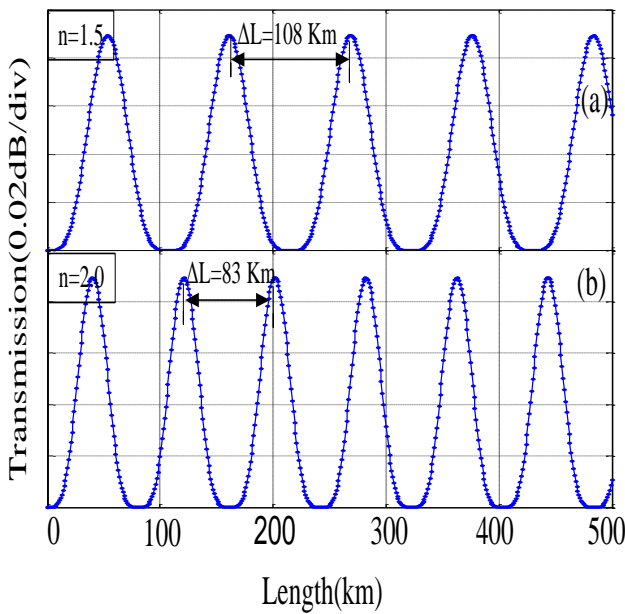

(a)

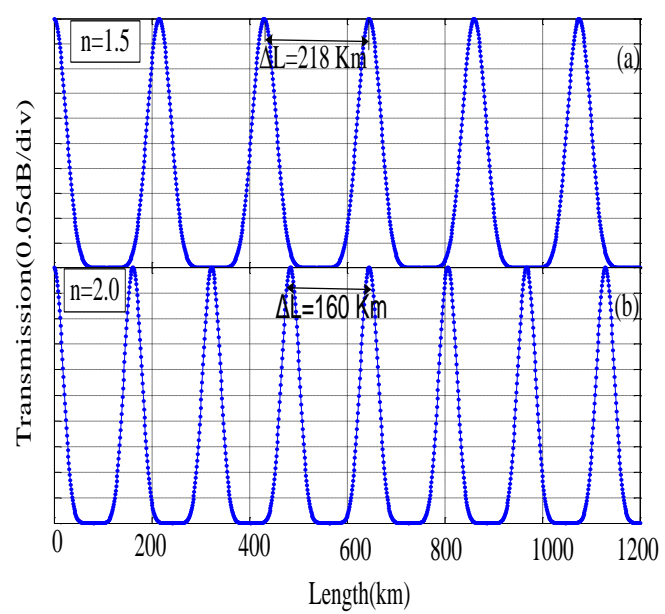

(b)

Fig 4: Transmission spectra for the variation of length. (a) $\varnothing=90^{\circ}$; (b) $\varnothing=45^{\circ}$ or $0^{\circ}$

The spectral spacing can also be changed by varying the length of the fiber. The variation of spectral spacings with the variation of fiber lengths based on equation (8) and (9) have been shown in figures 4(a) and 4(b) respectively. In figure 4(a), the peak to peak distances of $108 \mathrm{~km}$ and $83 \mathrm{~km}$ are obtained for the same fiber length using the refractive indices of 1.5 and 2.0 respectively. In figure 4(b), for a fixed fiber length of $1200 \mathrm{~km}$ the spectral spacings are found to be changed from $218 \mathrm{~km}$ to $160 \mathrm{~km}$ by changing the refractive index of the fiber material from 1.5 to 2 . In both cases, spectral spacing has been decreased with the increase of refractive index of the fiber material.

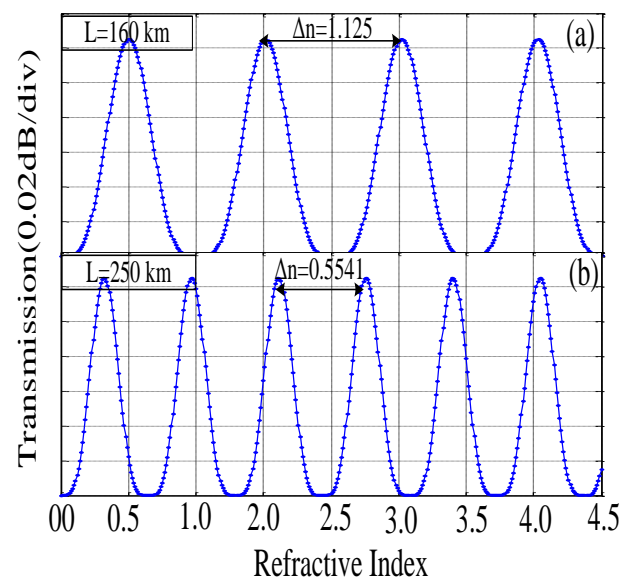

(a)

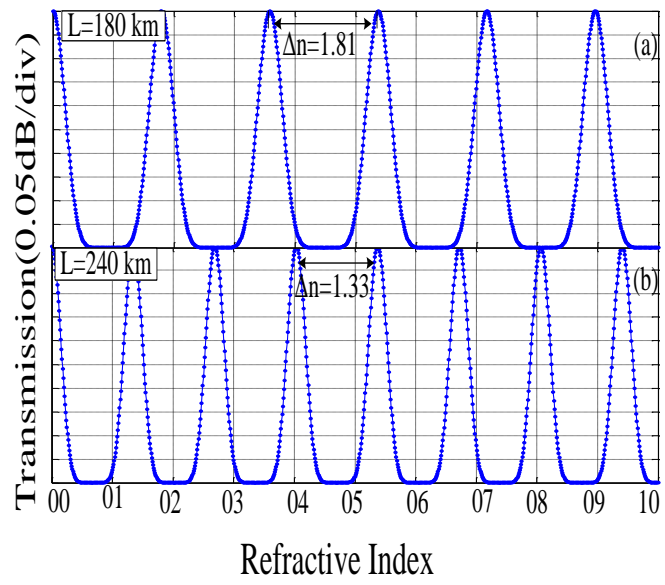

(b)

Fig 5: Transmission spectra with the variation of refractive index (a) $\varnothing=90^{\circ}$; (b) $\varnothing=45^{\circ}$ or $0^{\circ}$

Refractive index itself is a function of spectral spacing. Spectral spacing has been changed by changing the refractive index of the material finally. In figures 5(a) and 5(b) transmission pattern are shown for two different fiber lengths with the variation of refractive index. Figure 5(a) represents the output transmission spectrum for the variation of refractive index based on equation (8). We observed that refractive index of a material can be changed by changing its length. In figure 5(a), for the change of material length from $160 \mathrm{~km}$ to $250 \mathrm{~km}$ refractive index changes from 1.125 to 0.5541 . Output transmission spectrum based on equation (9) is shown in figure 5(b). In this case, refractive index has been changes from 1.81 to 1.33 by changing fiber length from $180 \mathrm{~km}$ to $240 \mathrm{~km}$. In both cases, refractive index decreases with the increase of fiber lengths. We consider a gradded index fiber in which refractive index decreases with the increase of fiber length. Thus we can have required number of peaks by increasing or decreasing the fiber length.

\subsection{Spectral Spacing Variation}

Spectral spacing is a function of wavelength of the input laser signal, fiber length, and refractive index of the fiber material. It also depends on the phase difference due to path difference between two interferometer arms. We have studied the variation of spectral spacing with different parameters keeping the phase difference 
$(\varphi)$ at some specific values. Figures showing the relationship of variation of spectral spacing with the variation of wavelength, refractive index and fiber length are discussed below in brief.

\subsubsection{With Wavelength Variation}

Variation of spectral spacing with the variation of wavelength based on equation (10) has been shown in figure 6. In equation (10) spectral spacing $(\Delta \lambda)$ is directly proportional to the square of the signal wavelength $(\lambda)$. It's a nonlinear relationship between them which is shown in figure 6 . Here, wavelength $(\lambda)=1550 \mathrm{~nm}$ and refractive index of the fiber material $n=1.5$ have been considered. It is observed that the laser signals exhibit the same behavior for different fiber lengths i.e. for $\mathrm{L}=200 \mathrm{~km}$ and $\mathrm{L}=410 \mathrm{~km}$.

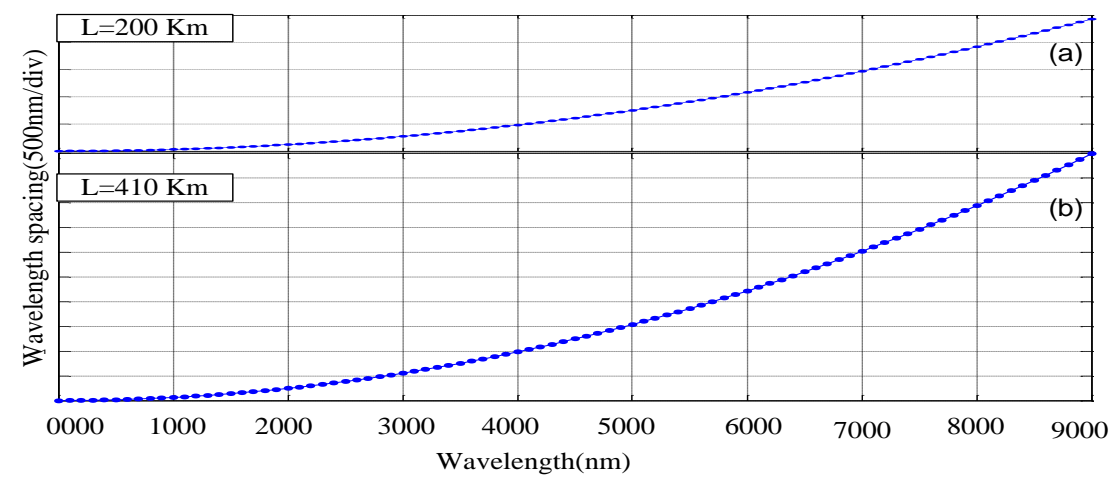

Fig. 6: Spectral spacing variation with the variation of wavelength [(a) $\mathrm{L}=200 \mathrm{~km}$ and (b) $\mathrm{L}=410 \mathrm{~km}]$

The non linear increment of spectral spacing with the linear increase of wavelength is observed from the above figure. Having refractive index (n) fixed if it is kept increasing of the signal wavelength $(\lambda)$, the spectral spacings $(\Delta \lambda)$ will be increased obeying the relationship $\Delta \lambda \propto \lambda^{2}$ for any length of the fiber.

\subsubsection{With Fiber Length Variation}

According to equation (10) spectral spacings are being decreased with the increase of fiber length. Thus the variation of spectral spacing with the variation of fiber length has been shown below in figure 7 . Figure 7(a) and 7(b) show the variation of spectral spacing with the variation of fiber length having two different refractive indices i.e. $\mathrm{n}=1.5$ and $\mathrm{n}=2.5$ respectively. Here wavelength of the laser signal $(\lambda)=1550 \mathrm{~nm}$ has been used. It is shown that spectral spacing changes in similar manner for both of the fiber materials.

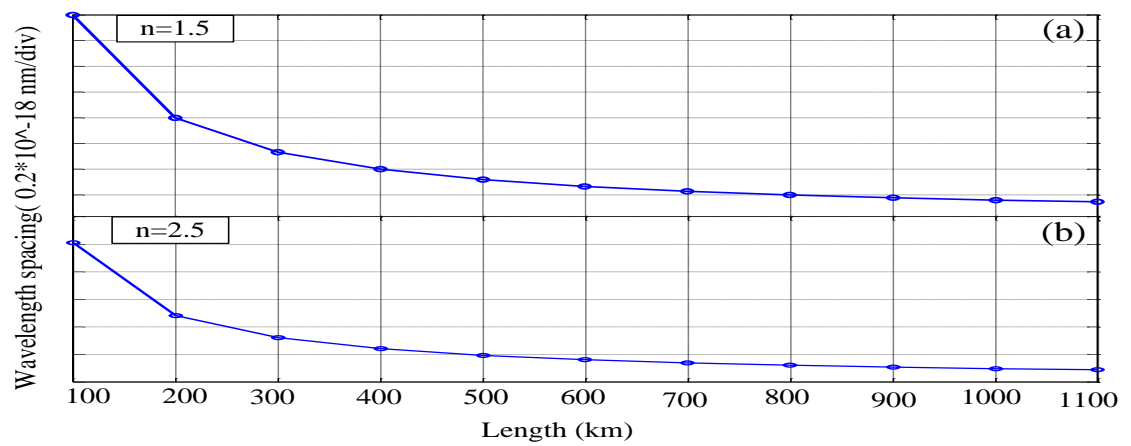

Fig 7: Spectral spacing variation with the variation of fiber length [(a) $n=1.5$ and (b) $n=2.0]$

From the above figure it is observed that spectral spacings decreases rapidly when the fiber length increased from $100 \mathrm{~km}$ to $300 \mathrm{~km}$. Spectral spacing remains almost constant for the change of length from $\mathrm{L}=600 \mathrm{~km}$ onwards.

\subsection{3with Refractive Index Variation}

Spectral spacing change with the variation of refractive index in a similar manner as they change with the variation of fiber length. Figure 8 shows the variation of spectral spacing with the variation of refractive index of the fiber material having two different fiber lengths. 


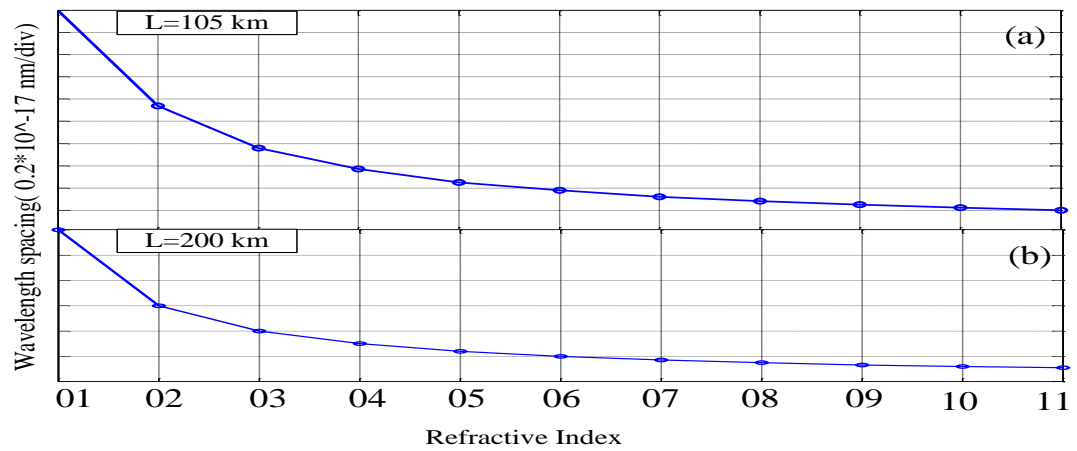

Fig 8: Spectral spacing variation with the variation of refractive index [(a) $\mathrm{L}=104 \mathrm{~km}$ and $(\mathrm{b}) \mathrm{L}=200 \mathrm{~km}]$

It is observed that in both cases, spectral spacing decreases rapidly for the change of refractive index from $n=1$ to $n=4$. Spectral spacings remain almost constant for the change of refractive index from $n=6$ to above. So there is a limitation of changing wavelength spacing with refractive index.

\section{Conclusion}

A new type of comb filter based on polarization maintaining fiber has been demonstrated and its operation in spectral spacing tunibility has been studied numerically. Continuous tuning of the spectral spacing can be obtained with simple adjustment of refractive index, fiber length and wavelength of the input signal. Graphs show that channel spacing can be increased or decreased by varying refractive index, fiber length and wavelength of the input signal. With our proposed comb filter we can adjust the waves to interfere with one another to ensure maximum transmission. The unique property of this filter is the polarization insensitivity. This comb filter is also expected to be useful to determine the specific tuning range of the output spectrum which is very important in WDM system. The proposed comb filter may find applications in generating tunable fiber laser as well as tunable multiple fiber laser sources. Finally, it is expected to find use in fiber sensor technology.

\section{References}

[1] S. Hu, L. Zhan, Y. J. Song, W. Li , S. Y. Luo and Y. X. Xia ,Switchable multiwavelength erbium-doped fiber ring laser with a multisection high-birefringence fiber loop mirror, IEEE Photon. Technol. Lett., 17( 7),1387 -1389, 2005

[2] Z. C. Luo, W. J. Cao, A. P. Luo, and W. C. Xu, Polarization Independent,Multifunctional All-Fiber Comb Filter Using Variable Ratio Coupler-Based Mach-Zehnder Interferometer,J. Lightw.Technol., 30(12), 1857-1862, 2012

[3] J. Stone, L. W. Stutz, and A. A. M. Saleh, Three-mirror fiber Fabry-Perot filters of optimal design,Electron. Lett., 26(14), 10731074,1990

[4] B. Lyot, Optical apparatus with wide field using interference of polarized light, C.R. Acad. Sci. (Paris), 197, 1933

[5] B. J. Eggleton, P. A. Krug, L. Poladian, and F. Ouellette, Long periodic superstructure Bragg gratings in optical fibers, Electron. Lett.,30( 19), 1620-1622, 1994

[6] Z. Zhang, L. Zhan, K. Xu, J.Wu, Y. Xia, and J. Lin, Multiwavelength fiber laser with fine adjustment, basedon nonlinear polarization rotation and birefringence fiberfilter," Opt. Lett., 33, 324-326, 2008

[7] X. Shu, S. Jiang, and D. Huang, Fiber grating Sagnac loop and its multiwavelength-laser application,IEEE Photon. Technol. Lett.,12, (8), 980-982, 2000

[8] H. L. An, X. Z. Lin, E. Y. B. Pun, and H. D. Liu, Multi-wavelength operation of an erbium-doped fiber ring laser using a dual-pass Mach-Zehnder comb filter,Opt.Commun., 169, 159-165, 1999

[9] Y. W. Lee, H. T. Kim, J. Jung, and B. Lee, "Wavelength-switchable flat-top fiber comb filter based on a solc type birefringence combination," Opt. Express, 13, 1039-1048, 2005

[10] H. Dong, G. Zhu, Q. Wang, H. Sun, N. K. Dutta, J. Jaques and A. B.Picciril, "Multi-wavelength fiber ring laser source based on a delayed interferometer," IEEE Photon. Technol. Lett., 17, 303-305, 2005

[11] Zhi-Chao Luo, Ai-Ping Luo, and Wen-Cheng Xu, Polarization-controlled tunable all-fiber comb filter based on a modified dualpass Mach-Zehnder Interferometer,IEEE Photon. Technol. Lett., 21( 15), 1066-1068, 2009 